\title{
Sustainable supply network structure: Understanding impact of inter- firms' relations as drivers of supply network complexity
}

\author{
Lokhman Hakim Osman ${ }^{1}$, Siti Norida Wahab ${ }^{2}$, Ahmad Raflis Che Omar ${ }^{1}$, Suraiya Ishak ${ }^{3}$, Azhar \\ $\mathrm{Ahmad}^{1}$
}

${ }^{1}$ Fakulti Ekonomi dan Pengurusan, Universiti Kebangsaan Malaysia

${ }^{2}$ UCSI University Malaysia

${ }^{3}$ Program Sains Pembangunan, Pusat Kajian Pembangunan, Sosial \& Persekitaran,

Fakulti Sains Sosial dan Kemanusiaan, Universiti Kebangsaan Malaysia

Correspondence: Azhar Ahmad (email: azah@ukm.edu.my)

Received: 01 March 2020; Accepted: 15 May 2020; Published: 30 May 2020

\begin{abstract}
This study examined the Inter-Firm Relation (IFR) as one of the elements to enhance Sustainable Supply Network Structure (SSNS) management in Malaysian maritime industry supply network. IFR has been an interesting research area for academics and businesses practices due to its relevance in determining the best practices and impacts to encourage SSNS. The Social Network Analysis (SNA) method was adopted to develop valid attribute for the measurement process and the embeddedness theory was used to evaluate IFR among the proposed attributes. These methods enable the researcher to transform relational data in the form of network matrix and produce reliable results for theoretical and industrial applications which have not been discovered in previous studies. A total of 37 managers in a maritime industry supply network were approached to participate in this study. Result revealed that IFR has different effects on the formation of SSNS. The finding provides useful insight in formulating the importance of IFR towards improving and understanding SSNS, particularly, in the Malaysian maritime industry. The contribution of this research extends to the literature and put forward solutions for the industry since previous studies are neglecting IFR, which is highlighted as a source of supply network complexity.
\end{abstract}

Keywords: complexity, inter-firm relation, social network analysis, supply network, sustainable supply network

\section{Introduction}

Development of a Sustainable Supply Network Structure (SSNS) has been a major concern for many firms (Nair et al., 2018). It is one of the main issues associated thru the complexity within the supply network. Supply network complexity (SNC) has been one of the major issues in regards to the development of SSNS. Specifically, it is the complexity that originate from the uneven, still widespread inter-firm relationships (IFR) exists amongst firms within the supply network. The complexity of the supply network structure (SNS) is becoming more 
difficult to understand due to the unique type of IFR that the firms are embedded in (Nair et al., 2018).

Furthermore, not all firms in the supply network are engaging in sustainable relational-based behaviour (Ceyhan et al., 2018). Numerous decisions and actions in a supply network were performed based on attributes of firms' analysis compared to the degree of IFR (Mohd Adnan \& Valliappan, 2019). This is due to the facts that attributes are more visible and easily evaluated compare to IFR. Inter-firms' relation was argued to be more tacit and subtle. Attributes of firms in the supply network can exist between firms because of the limit, culture, geological area and long stretches of activity and size and physical elements.

Firms' attributes are responsible for producing sensible and effective decision making. As expressed by Chakkol et al. (2018a) and Osman (2018), firms must enlighten another important element responsible for the supply network sustainability (i.e. IFR). In previous studies, the complexity of the supply network being addressed as related attributes of the firms that make the level of separation among firms (Turner et al., 2018). The debate is that, as the quantity of firms inside the supply network has been built, this will expand the administrative and operational necessities which expected to deal with the distinctive attributes with different firms.

However, attributes are not the only reasons organizations are embedded in the network. Aside from attributes of firms, SSNS also define as a result of the degree of IFR within the supply network (Chakkol et al., 2018b). Research in the area of supply chain and operations management contribute to IFR as ties among the firms from the system structure. IFR inside SSNS may be inside the setting of trades or information trades. For instance, information data or contractual related streams. What makes IFR more difficult to manage because of IFR may exist in supply as part of the formal network connections (contractual obligations) or informal network connections (information sharing), nonetheless the knowledge of the existence only applicable to the connected few networks. Thus, supply network management requires an improved proposition when dealing with supply network complexity. Because of the complexity of IFR, only a few pieces of research relate the subject of SSNS with IFR were found. Therefore, there is a need to assess IFR to improve understanding of SSNS. This research proposes to enlighten IFR as one of the elements to enhance SSNS management.

The objective of this study is to assess IFR that are related to SSNS management among the maritime industry supply network in Malaysia. In recent years, very few studies associated with this topic being focus particularly in Malaysia's market. Mutke et al. (2019) in their study described SSNS as hourglass-shaped. It is defined as numerous different stakeholders at the supply and demand end whereas in the middle consist of small number of trading companies. Most of the members of the supply network saw numerous and various partners of the supply network which representing a significant obstruction to the usage of duties by clouding recognisability and ruining commitment with clients or providers. Meanwhile, this research analyses the supply network not from the attribute context of the firms but IFR context. Specifically, this research extends to enrich the current literature and recommend solutions to the industry players since previous studies are neglecting the importance of IFR elements. 


\section{Literature Review}

\section{Sustainable supply network structure}

Sustainable supply network structure (SSNS) describe as the multifaceted nature that emerges from the connectivity among the embedded components in the SNS towards accomplishing accord sustainable objectives (Osman, 2017). These portrayals of multifaceted nature would legitimize the contention that SSNS is likewise complex and IFR speaks to the interconnectivity between the components in the structure. Hartman (2016) allude to complexity in the network as to how much network members interrelate. IFR between firms in SSNS could be as physical merchandise trades or data trades, sustainable information or contracts regulations (Hartman, 2016; Osman, 2017).

With regards to SSNS, Chakkol et al. (2018a) and Chakkol et al. (2018b) have expressed that SSNS have been encountering expanded multifaceted nature through broad IFR. For instance, in SSNS of vehicle producers, a provider may supply parts to a maker, while this maker may simultaneously supply different parts to a similar provider organization. Most importantly the considerable lot of these working relations among firms in SSNS frequently exist and past the knowledge of the central firm. Therefore, this creates difficulties in ensuring and maintaining green practices among the embedded firms of SSNS.

A firm within SSNS may accept IFR if the data traded advances better coordination of SSNS (Hamari et al., 2016). Notwithstanding, it might be unwelcome if IFR adds to spillage the data in SSNS. Thus, an association's observations concerning a decent accomplice may change unexpectedly. In this manner, IFR among firms in SSNS is a significant part of SNC. IFR in SSNS (regardless of whether the organizations contend or collaborate) has been found to essentially affect the value proposition of the central firm (Kim et al., 2015a).

Another stream of research shows that the development of SSNS makes an increasingly complex structure emerging from the IFR (Kim et al, 2015b). What makes SSNS increasingly complex is due to the current examinations have just been concentrating on the prescribed materials stream sort of IFR amongst the firms in SSNS. A different type of IFR which added to the general multifaceted nature in SSNS has been identified. This is because, as shown by the embeddedness hypothesis and the investigations, the conventional business exchanges in SSNS are inserted in a web of informal social exchanges (Chakkol et al., 2018a)

IFR adds to expanding unpredictability in SNC adding to the precursors of SSNS. Kim et al. (2015b) have inferred that IFR is one of the facets of the complexity in SSNS and more profound comprehension is expected to beat the complexity nature coming about because of these IFR. All the more explicitly, the diverse IFR between them makes the degree of complexity which requests effective administration techniques from supervisors (Osman et.al. 2017). These various causes of complexity request a reasonable clarification of SSNS for compelling and proficient administration of the supply network.

In this way, understanding SNC means understanding the relations among firms in SSNS. This examination considers SNC as connections which are clarified through how IFR emerges among firms in SSNS. With the idea, there are featured IFR, for example, formal between firm relations (FIFR) and informal between firm connection (IFFR). Hence, this examination proposes to improve the literature on SSNS and SNC by utilizing those IFR to evaluate.

Numerous variables of SSNS have likewise been looked into and examined. Despite the measure of earlier research and the components considered, there is still a new requirement for new investigations to satisfy the total cognizance of SSNS. Increasingly exhaustive investigations are as yet should have been performed with the goal that the consciousness of SSNS can be elevated both in the scholarly world and industry. In this 
investigation, the researcher features two components to help the research fundamental measurement. Moreover, the embeddedness theory contends that these connections or IFR can be as formal business exchanges exercises, for example, legally binding relations or of casual social trades, including data sharing and referral exercises (Papadonikolaki et al, 2017). These kinds of IFR can be either integral or substitutes of the other. Thus, an increasingly exact understanding of SSNS is required.

\section{Inter-Firm Relations (IFR)}

Fundamentally, SNC of SSNS is shaped by the network or connections between firms where the combination logically frames a definitive structure, which is simply SN. The connections are additionally referred to in the literature as IFR (Chakkol et al., 2018a; Chakkol et al., 2018b). An IFR represent two hubs (or dyad) and one connection in a network term. Every hub can be hypothesized as an actor performing certain activities to produce a high value. The firm needs assets from its provider firm, and the provider needs agreements and instalments from the purchaser. Contracts make the SN's Formal Inter-Firm Relation (FIFR) (Chakkol et al., 2018a). FIFR directly affects SNC through ties availability. By formal authoritative relations, the central firms authorize formal relations through legally binding terms (FIFR) and guidelines upon other network members. Over that FIFR, firms' interface to share data concerning advertising openings and new dangers (Cousins et al., 2006). As an outcome, these create a connection and structure IFR or dyad. Since a firm frequently has connections to different firms, the firm brings to the dyad new by implication associated firms. Thus, with the provider firm, this will likewise carry to the dyad their connections with different firms either legitimately or in an indirectly way (Choi and Kim, 2008). Indisputably, a FIFR is a dyad; it is additionally part of a network.

Furthermore, within an SSNS, IFR may also take the form of informal information sharing ties (IIFR) (Chen et al., 2018; Šümane et al., 2018; Kim et al., 2015a). Lu and Huang (2018) acknowledged the above-mentioned ties based on the five types of establish relationships namely, (i) short term trade; (ii) semi and long-term trade; (iii) coordinatedprofit sharing; (iv) long term alliance; and (v) joint venture. Short-term trade indicates the transaction after the relationship ends. Meanwhile, semi and long-term trade indicates the trade agreements beyond a formal contract that legally bind within the firms. Notably, it also involves informal forms of relations (IFFR), akin with other commercial connections including information sharing and referral activities. All of this creates a momentous firm competitive advantage who embedded in such relationship formation.

SSNS is a complex concept that can be explained with various dimensions. Prior studies have tried to explain SSNs and proposed solutions through merely attribute dimensions. In the context of IFR, it can be seen that those correlated parties which involved directly in the multi-faceted nature of IFR encountered network relationships conflicts. Subsequently, former works of literature also light up multiple issues about IFR complexity in SSNS. Two types of IFR has been recognized contributed to the pattern of firm embeddedness over its network structural positions namely the contractual tie (FIFR) and the information sharing tie (IIFR). This study uses the dimensions of IIFR and FIFR that can best fulfil the research objective.

\section{Methodology}

An interrelated firm working together in a maritime industry formed the sample of this research. The focal firm in this study is the APMMHQ-1 (nom de plume for the motivations 
behind secrecy). The APMMHQ-1 is a shipbuilding assembling organization in the Malaysian industry engaged with ship fixes, sea works and building. Until this point, the organization has granted agreements to nearby merchants and providers totalling RM31 million for the improvement of little vessels in the locale.

\section{Social Network Analysis Method}

Wasserman and Faust (1994) confront that the standard statistical technique and investigation isn't proficient in estimating relations. There is a standard statistical investigation disclaims the presence of the connections amongst firms in a network over the suspicion of freedom and perception. The Social Network Analysis (SNA) enable to centres the relations amongst firms and the ramifications of the connections. Organizing of network of relations has a significant ramification for the firms in a different network. For this study, exploratory network analysis has been applied to investigate various collaborations among firms which used to enlighten the general example of embeddedness of firms in the APMMHQ-1 SSNS. This examination was performed by utilizing the social network software, which includes UCINET, NetDraw, Mage and, Pajek (Borgatti et al., 2002). Following are the explanatory steps of SNA for this investigation.

\section{Data Analysis}

\section{Determine the study population}

The initial step of SNA is to decide the number of populations of investigation. This study has identified two units of analysis which is the firms that pattern the APMMHQ-1 SSNS, and the ties between them. In the network study, the strategy used to test relations is a piece of the study instrument. In this investigation, the analyst applied the pragmatist and nominalist approach to decide the suitable study sample.

\section{Network data collection}

This study developed a survey form which consists of both closed-ended questions and openended questions. The survey starts with the general questions pursue with the additional specific and inquiring questions. The network survey form was adopted from various network queries of former network studies on IFR (Borgatti \& Li, 2009; Borgatti et al., 1998; Cousins et al., 2006; Krause et al., 2007)

One class of queries examines the network ties relationship amongst the corporations within the APMMHQ-1 network. During this exercise, the survey displays a table with the names of all the corporations listed within the initial column of the table. To support this exercise, the respondents were asked to provide a point by a checked on the table of the list provided. It is a list of a corporation that they have been in touch in the past sixth months for every type of IFR. These IFR are necessary to grasp each formal and informal relationship amongst them. The kinds of ties were investigated specifically on the contracts and information sharing. The survey instrument intentionally asked the key informants to point out the list of firms that they required for the formal green service contracts which concerning the provision of materials for the merchandise RHIB. 


\section{Degree of connectivity index}

Density being outlined because the variety of connections of the participant has divided by the overall potential connections that the participant might have. For instance, if twenty folks are collaborating, every person might doubtless hook up with nineteen people. A density of 100 per cent (19/19) is the greatest density within the system. A density of fifty indicates there are just one of nineteen potential connections among the SNS. Network density measures the strength of the linkages or connection among actors in a network. Based on Wasserman and Faust (1994), a network density is determined by dividing the number of actual ties amongst the network actors with the number of potential ties as defined in equation 1 .

$$
\text { DENSITY }=\frac{2 L}{g(g-1)}
$$

where $L$ serves as the number of lines present in the network and $g$ serve as the number of nodes recorded in the network. The common report for the density scores is in the percentage form. The advanced the density of the network, determine the more connected the network are. Thus, it can conclude that a density score of 1 represents an absolute network connection among all actors in the network through a certain type of ties. Contrarily, a density score of 0 represents entirely fragmented network with no ties testified between the actors.

\section{Pattern of connection index}

The second index is the centralization index. According to Freeman (1979), the network centralization index measures the degree to which the network is centralized around a single or few actors in the network. It measures the degree that network members interact with others within the network. Freeman (1979) in his study determine the index of centralization is described from the ratio of the sum of the actual variance between the centrality of the most central point, and that of all the other points and the highest possible sum of variances in actor centrality as prescribe in equation 2 .

$$
\text { CENTRALIZATION } \frac{=\sum_{i=1}^{g}[\operatorname{Ca}(n *)-\operatorname{Ca}(n i)]}{\max \sum_{i=1}^{g}[\operatorname{Ca}(n *)-\operatorname{Ca}(n i)]}
$$

where $\mathrm{Ca}(n i)$ and $\mathrm{Ca}\left(n^{*}\right)$ are an actor centrality index and the largest value of the particular index that occur across the $g$ actors in the network respective (Freeman, 1979). The value of the network centralization differs between 1 and 0 , where, a advanced value specifies greater network centralization around an actor of few actors.

\section{Mapping the causal IFR diagram}

In the analysis, we also provide the network plots of the different relationships focusing on firms. This analysis is done using NetDraw and Mage. The generated network plots will fully capture the research question as it will graphically imply the extent of embeddedness of firms in the network base on the different type of relationships. 


\section{Results and Discussion}

\section{The density index score}

In Table 1, the first column represents the density scores of the ties or linkages. Due to each tie in this study generates respective matrices, the density scores in Table 1 is the density score for each of the contractual ties and information sharing ties. Based on Table 1, we could see that among IFR density, the information-sharing ties (IIFR) has the highest density score of 0.2965 . It is followed by contractual ties (FIFR) density with 0.1660 . The density scores illustrate that there is more information sharing ties SNS than there are contractual tie linkages between the member firms. This also means that in SSNS of the maritime industry in Malaysia the IIFR shows more weight or importance compared to the FIFR.

Table 1. SSNS Density index score

\begin{tabular}{ccc}
\hline Type of Linkages & Density & IFR Continuum \\
\hline Contractual & 0.1660 & FIFR \\
Information Sharing & 0.2965 & IIFR \\
\hline
\end{tabular}

A result of this study reveals a consistency outcome which is akin to other researcher findings in other fields of inter-firm studies (Cousins et al., 2006; Oh et al., 2004). It is claimed that less formal interactions occur rather frequently among firms with information gathered from the informal ties is more straightforward compared to formal ties. Moreover, the standard collaboration amongst forms able to minimizes the requirements for the formal ties. This is due to the informal collaboration exemplify as a yardstick rather than exclusion. Thus, firms are often not involved with any informal ties with multiple types of firms which usually took place in the form of contracts or transmittal of funds.

\section{The centralization index score}

Table 2 exhibit the centralization index score of the linkage matrices. The centralization index for the contractual ties is 0.31428 and the centralization score for information sharing is 0.4724. This score suggests that FIFR such as contract ties are less centralized. It is common in a centrally managed system such as SSNS to have the focal firms to closely monitor the activities within which the network makes up.

Table 2. SNS centralization score

\begin{tabular}{ccc}
\hline IFR & Network Centralization & IFR Continuum \\
\hline Contractual & 0.3142 & FIFR \\
Information Sharing & 0.4724 & IIFR \\
\hline
\end{tabular}

The information sharing linkages centralization score is 0.4724 . Although previous studies in other contexts such as healthcare firms have found that the information sharing centralization index displays a very low score, this study found that in the context of the SNS, the centralization index remains high. One of the reasons is because, in SNS, the focal firms mandate the flow of resources within a network. Thus, it leads to the other firms seeking the information directly or indirectly from the focal firms. It can be in the form of orders and supply on a perpetual basis. This scenario makes the focal firms as a centre of information sources, consequently, provide the focal firms with a dynamic competitive advantage. 


\section{Description of Network Plot}

Figure 1 model a network map of SSNS with the continuous attribute degree centrality generated employing the spring embedding algorithm. As shown in Figure 1, each firm is identified by square shapes with lines indicating FIFR tie between the network members. Node size in Figure 1 is categorized based on the level of degree centrality in FIFR. The higher the degree centrality of the network members in FIFR, the bigger will be the size of the nodes. Principally, we could see in Figure 1 that nodes are highly involved or embedded in FIFR using degree centrality as the parameters are central in SSNS network. It is evident in Figure 1 that nodes like APMMHQ-1, MTURAWNG-3, MTUPJAYA-2, MTUKTAN-24 are all highly embedded in FIFR network are also positioned centrally in the trusted network. These core members of the network are highly connected among each other forming dense cohesive subsets, whereas a peripheral member appears to be more distant (e.g., PMMRSNG17, DMSDILI-16, DMKNTAN-21).

\begin{tabular}{|l|l|}
\hline Organization Code & $\begin{array}{l}\text { Contract } \\
\text { Tie/FIFR }\end{array}$ \\
\hline APMMHQ-1 & 30.000 \\
\hline MTUPJAYA-2 & 30.000 \\
\hline MTURAWNG-3 & 33.000 \\
\hline WILUTA-4 & 14.000 \\
\hline DMLKAWI-5 & 15.000 \\
\hline DMPPINANG-6 & 12.000 \\
\hline DMLUMUT-7 & 13.000 \\
\hline PMKKEDAH-8 & 12.000 \\
\hline PMKKURAU-9 & 10.000 \\
\hline PMKPERLIS-10 & 12.000 \\
\hline MTUPINANG-11 & 14.000 \\
\hline WILSEL-12 & 17.000 \\
\hline DMJBARU-13 & 11.000 \\
\hline DMPKLNG-14 & 10.000 \\
\hline DMKLGGI-15 & 10.000 \\
\hline DMSDILI-16 & 8.000 \\
\hline PMMRSNG-17 & 10.000 \\
\hline PMBPAHAT-18 & 11.000 \\
\hline MTUJB-19 & 32.000 \\
\hline WILTIM-20 & 11.000 \\
\hline DMKNTAN-21 & 0 \\
\hline DMKGANU-22 & 0 \\
\hline DMTBALI-23 & 0 \\
\hline MTUKTAN-24 & 32.000 \\
\hline WILSAR-25 & 15.000 \\
\hline DMKCHNG-26 & 11.000 \\
\hline DMBTULU-27 & 10.000 \\
\hline DMMIRI-28 & 10.000 \\
\hline PMTMANIS-29 & 9.000 \\
\hline MTUKCHG-30 & 8.000 \\
\hline WILSAB-31 & 16.000 \\
\hline DMLBUAN-32 & 11.000 \\
\hline DMKBALU-33 & 12.000 \\
\hline DMSDAKAN-34 & 10.000 \\
\hline DMTAWAU-35 & 0 \\
\hline PMLDATU-36 & 10.000 \\
\hline MTUKBALU-37 & 0 \\
\hline
\end{tabular}

Figure 1. Network Map Based on Degree Centrality in contract tie FIFR 
Similarly, evidence in Figure 1 demonstrated how the firms with high embeddedness score in FIFR (grounded on the degree centrality) are located centrally in SSNS. For example, APMMHQ-1, MTUKTAN-24, MTURAWNG-3, MTUJB-19 and MTUPJAYA-2 are located rather central in SSNS. Observing the degree centrality index score, APMMHQ-1, MTUKTAN-24, MTURAWNG-3, MTUJB-19 and MTUPJAYA-2-degree centrality score in the FIFR are 30.000, 33.000, 32.000, and 30.000 respectively. Also evident in Figure 1 is how the firms that are low in embeddedness degree in FIFR are situated or located far out in the trusted network. For instance, MTUKBALU-37, DMTBALI-23, DMTAWAU, DMKNTAN-21 are peripherals in SSNS with embeddedness score of zero for each one of them. Other firms such as WILSAB-31, WILUTA-4, MTUPNANG-4, WILSEL-12, and PMLDATU-36 are represented by medium-size squares which indicate their medium level of embeddedness. WILSAB-31, WILUTA-4, MTUPNANG-4, WILSEL-12, and PMLDATU36 embeddedness level (grounded on the degree centrality in contract tie) are recorded to be $16.000,14.000,14.000,17.000$, and 10.000 respectively.

Next, the researcher examines the findings in another IFR (i.e. the information sharing/IIFR). As mentioned, information sharing tie is considered an informal form IFR (Lamming et al., 2000). Likewise, in this section, the researcher developed a network map using the NetDraw package. Alike, the network map will display SSNS with the firm's degree centralization attributes represented by the size of the nodes in the network. The size of the nodes will reflect the nodes attribute (i.e. embeddedness based on degree centrality in IIFR).

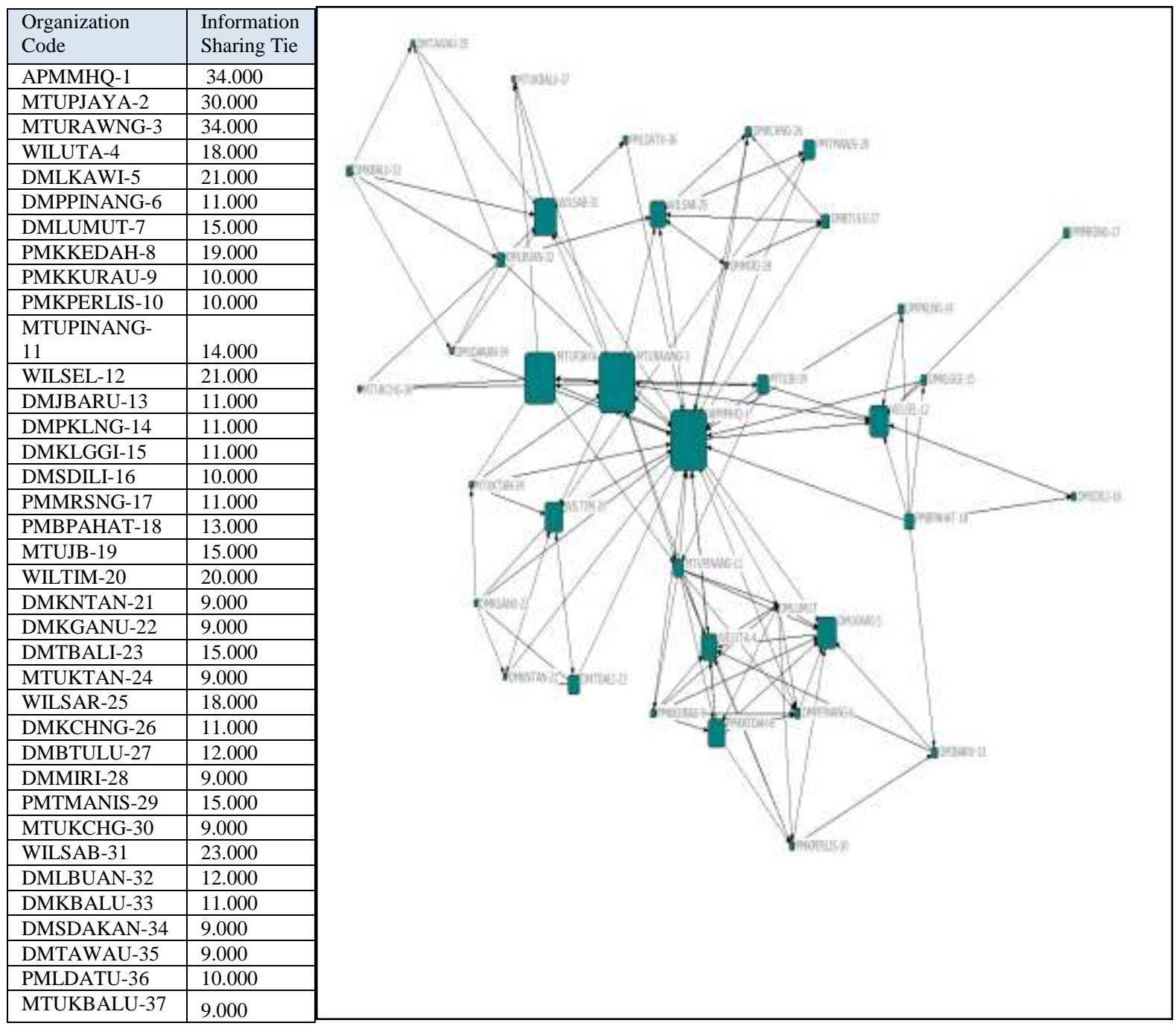

Figure 2. Network Map Based on Degree Centrality in information sharing tie IIFR 
Figure 2 demonstrated the sociogram displays SSNS with the attribute (i.e. embeddedness based on degree centrality in information sharing tie/IIFR). The embeddedness attributes are indicated by the sizes of the nodes in the figure. Based on Figure 2, several central nodes in SSNS which comprises of APMMHQ-1, MTURAWNG-3, MTUPJAYA-2 and WILSAB-31 can be confirmed. All these central nodes have a high embeddedness attribute based on degree centrality in IIFR. Although the number of central nodes with high embeddedness attributes is lower compare to the data generated in Figure 1, three of the central nodes or buyer-supplier organization of Figure 2 displayed a similar result as shown in Figure 1. APMMHQ-1 has embeddedness score of 34.000, MTURAWNG-3 has embeddedness attribute of 34.000, and MTUPJAYA-2 has an embeddedness score of 30.000. Besides, firms with medium size squares are located further out of the centre. These nodes possessed medium embeddedness score in IIFR, which means that their degree centrality score in IIFR ties are average. For example, firms such as WILSAR-25, WILSEL-12, WILTIM-20, DMLKAWI-5, WILUTA-4, MTUJB-19, PMKKEDAH-8 and MTUPINANG11 have embeddedness score of 18.000, 21.000, 20.000, 21.000, 18.000, 15.000, 19.000, and 14.000 respectively. Figure 2 also shows the nodes or buyer-supplier organizations that occupy the periphery location of the sociogram. In this case, the periphery nodes include firms such as MTUKBALU-37, DMTAWAU-35, MTUKCHG-30, MTUKTAN-24, DMKGANU-22, and DMKNTAN-21. These firms possessed some of the lowest embeddedness scores in the IFFR together with the positioning and size of the nodes of SSNS with the embeddedness (grounded on the degree centrality), as the nodes attributes indicate two important things.

Two aspects are prioritized in this study namely, Formal Inter-Firm Relation (Contract Relations) and Informal Inter-Firm Relation (Information Sharing Relation). From the exploratory network analysis of the network maps, a firm which is embeddedness in SSNS are associated to the degree of formality of IFR. Informal Inter-Firm Relation (Information Sharing) shows to have the strongest effect toward the aspect of IFR and consequently SSNS. Earlier studies emphasized the important role of IIFR in obtaining opportunities or innovations.

This study emphasizes that IIFR should be intensively managed by the firms embedded SSNS to tackle SNC because IIFR shows a strong effect on SSNS and consequently SNC. IIFR approaches through information sharing activities may generate more collaboration and interactions with other members in the SNS. IIFR efforts should emphasize information sharing activities. Therefore, the impact of IFR sticks more firmly with the members of the network and the resulting SNS. IFR also shows to have included FIFR. This could be because the basis on a SSNS usually comes from the FIFR before IIFR becomes abundance. What is means is that many IFR in SSNS is first forms because firms are embedded in SSNS through the contractual obligation of supply and distribution. Eventually, IIFR becomes more abundance as firms become more connected to other firms through other types of IFR including IIFR. On the theoretical level, it can be said that informal relations can be a better mechanism for the medium of communication in SSNS. The more firms can embrace the importance of IIFR, the more effective it may affect the management of SSNS and consequently SNC.

\section{Conclusion}

Topics about SSNS complexity have not been often associated with the role of IFR. Earlier studies often link SNC with attributes of the firms embedded in SSNS. This study highlights the importance of how firms interact with other firms in SSNS. Therefore, using the SNA 
method, these study asses a set of dimensions and elements to determine the importance of IFR for improving and understanding SSNS in the maritime industry in Malaysia. These methods enable the researcher to transform relational data in the form of network matrix and produce reliable results for theoretical and industrial applications which have not been discovered in previous studies.

The findings of this study contributed to the context of SSNS. Firms' which is embeddedness is built upon on the type of network relations thus, creating a new structure rather than the traditional linear structure of the supply network. The exploratory network analysis in this study has given a strong indication of SSNS. Extra attention and resources (as forming a new alliance entails time and even money) of the embedded firms are devoted mainly for the informal networks of relations rather than the formal networks. Overall, this study found that the context of SNS among a firms' embeddedness is depending upon the type of IFR. This study confirmed that firms are more embedded in informal networks than informal networks.

In consideration of the overall implications of this study, it can wrap up that supply network evolves. The managers required to scrutinize their firm's existing embeddedness towards the competitive advantage establishment among the supply network inter-firm relationships. Firms which fail to comprehend the foundations of these relationships are likely to encounter hitches within the network itself. Therefore, managers who intend to build a competitive advantage from the network should effectively engage with other partners. Some firms have shown adequate standing, while some are struggling in some areas. Future studies should replicate the framework of this study in a different geographical area. It also can be enforced by managers who are committed to engaging with other network associates.

There are some limitations to this study. First, this study is limited to the maritime industry, therefore not all the result can be generalized for all other industries and countries. Second, this study is limited to the information sharing ties as IIFR. Other IFFR that include referral relations may provide unique sets of findings. Future studies could use other types of IFR that include referral ties in other forms of industry. Further literature on SNC should be enriched depending on the specific relations that are has been used to be analysed in this study.

\section{Acknowledgement}

This work was supported by the Dana Cabaran Perdana Grant [DCP-2018-002/3]

\section{Reference}

Borgatti, \& Li. (2009). On social network analysis in a supply chain context. Journal of Supply Chain Management, 45(2), 5-22.

Borgatti, S. P., Jones, C., \& Everett, M. G. (1998). Network measures of social capital. Semantic Pajek Networks Software, 21(2), 27-36.

Borgatti, S., Everett, M., \& Freeman, L. (2002). UCINET 6 For Windows: Software for social network analysis. Retrieved from http://www.analytictech.com/

Borgatti, S.P. and Everett, M.G. (2000) Models of core/periphery structures. Social Networks, 21, 375-395.

Burt, R.S. (1995). Structural holes: The social structure of competition. Harvard University Press. 
Burton, P. (2010). Social network position and its relationship to performance of IT professionals. Informing Science: The International Journal of an Emerging Transdiscipline, 13, 121-137.

Ceyhan, S., Dogan, I.C., Yildiz, M., \& Barca, M. (2018). Boundary conditions for the emergence of relational trust among exporters and importers. Review of International Business and Strategy, 28(3/4), 358-372.

Chakkol, M., Finne, M., Raja, J.Z., \& Johnson, M. (2018a). Social capital is not for sale: A supply network perspective on mergers and acquisitions. Supply Chain Management: An International Journal, 23(5), 377-395.

Chakkol, M., Selviaridis, K., \& Finne, M. (2018b). The governance of collaboration in complex projects. International Journal of Operations \& Production Management, 38(4), 997-1019.

Chen, M., Liu, H., Wei, S., \& Gu, J. (2018). Top managers' managerial ties, supply chain integration, and firm performance in China: A social capital perspective. Industrial Marketing Management, 74, 205-214.

Choi, \& Kim. (2008). Structural embeddedness and supplier management: A network perspective. Journal of Supply Chain Management, 44(4), 5-13.

Cousins, P.D., Handfield, R.B., Lawson, B., \& Petersen, K.J. (2006). Creating supply chain relational capital: The impact of formal and informal socialization processes. Journal of Operations Management, 24(6), 851-863.

Freeman, L.C. (1979). Centrality in social networks conceptual clarification. Social Networks, 1(3), 215-239.

Hamari, J., Sjöklint, M., \& Ukkonen, A. (2016). The sharing economy: Why people participate in collaborative consumption. Journal of the Association for Information Science and Technology, 67(9), 2047-2059.

Hartman, S. (2016). Towards adaptive tourism areas? A complexity perspective to examine the conditions for adaptive capacity. Journal of Sustainable Tourism, 24(2), 299-314.

Hedvall, K., Jagstedt, S., \& Dubois, A. (2019). Solutions in business networks: Implications of an interorganizational perspective. Journal of Business Research, 104, 411-421.

Kilduff, M., \& Tsai, W. (2003). Social networks and organizations. Sage Publications Ltd.

Kim, J., Yoon, Y., \& Zo, H. (2015a). Why People participate in the sharing economy: A social exchange perspective. PACIS 2015 Proceedings, 76.

Kim, Y., Chen, Y.-S., \& Linderman, K. (2015b). Supply network disruption and resilience: A network structural perspective. Journal of Operations Management, 33, 43-59.

Kirchoff, J.F., Tate, W.L., \& Mollenkopf, D.A. (2016). The impact of strategic organizational orientations on green supply chain management and firm performance. International Journal of Physical Distribution \& Logistics Management, 46(3), 269-292.

Krause, D.R., Handfield, R.B., \& Tyler, B.B. (2007). The relationships between supplier development, commitment, social capital accumulation and performance improvement. Journal of Operations Management, 25(2), 528-545.

Lamming, R., Johnsen, T., Zheng, J., \& Harland, C. (2000). An initial classification of supply networks. International Journal of Operations \& Production Management, 20(6), 675691.

Lu, L.H., \& Huang, Y.F. (2018). Manufacturing strategy, organizational slack, and the formation of interfirm linkages. Chinese Management Studies, 13(1), 70-92. DOI: https://doi.org/10.1108/CMS-08-2017-0238

Mohd Adnan, S.N.S., \& Valliappan, R. (2019). Communicating shared vision \& leadership styles towards enhancing performance. International Journal of Productivity and Performance Management, 68(6), 1042-1056 
Mutke, S., Bonet, J.A., Calado, N., Calvo, J., Taghouti, I., Redondo, C., \& Martinez de Arano, I,, (2019). Innovation networks on Mediterranean non wood forest products. Journal of Innovative Science and Engineering, 3(1), 1-10

Nair, A., Blome, C., Choi, T.Y., \& Lee, G. (2018). Re-visiting collaborative behavior in supply networks-structural embeddedness and the influence of contextual changes and sanctions. Journal of Purchasing and Supply Management, 24(2), 135-150.

Oh, H., Chung, M., \& Labianca, G. (2004). Group social capital and group effectiveness: The role of informal socializing ties. The Academy of Management Journal, 47(6), 860-875.

Osman, L.H. (2017). The pattern of inter-organizational level of connectivity, formal versus informal ties. Jurnal Komunikasi: Malaysian Journal of Communication, 33(1), 59-79.

Osman, L.H., 2017. Organizational embeddedness and its effect on performance: An exploratory analysis for Malaysian business managers. Geografia-Malaysian Journal of Society and Space, 12(2), 212-222.

Osman, L.H. (2018). Embeddedness configuration and its' relations to inter-firm connectivity in supply network: A social network analysis approach. Journal of International Trade, Logistics and Law, 4(1), 31.

Papadonikolaki, E., Verbraeck, A., \& Wamelink, H. (2017). Formal and informal relations within BIM-enabled supply chain partnerships. Construction management and economics, 35(8-9), 531-552.

Provan, K.G., \& Kenis, P. (2008). Modes of network governance: Structure, management, and effectiveness. Journal of Public Administration Research and Theory, 18(2), 229.

Šūmane, S., Kunda, I., Knickel, K., Strauss, A., Tisenkopfs, T., Rios, I.I.D., Rivera, M, Chebach, T \& Ashkenazy, A. (2018). Local and farmers' knowledge matters! How integrating informal and formal knowledge enhances sustainable and resilient agriculture. Journal of Rural Studies, 59, 232-241.

Turner, N., Aitken, J., \& Bozarth, C. (2018). A framework for understanding managerial responses to supply chain complexity. International Journal of Operations \& Production Management, 38(6), 1433-1466.

Von Danwitz, S. (2018). Managing inter-firm projects: A systematic review and directions for future research. International Journal of Project Management, 36(3), 525-541.

Wasserman, S., \& Faust, K. (1994). Social network analysis: Methods and applications: Cambridge University Press. 